\title{
Review of: "Dynamics of cortical oxygenation during immediate adaptation to extrauterine life"
}

\author{
Gorm Greisen ${ }^{1}$ \\ 1 Capital Region of Denmark
}

Potential competing interests: The author(s) declared that no potential competing interests exist.

Nicely confirming expectations and previous work: The brain pink-up within a few minutes after birth, from a tissue oxygenation value of perhaps $50 \%$ in the fetal state to around $75 \%$ within the first 10 minutes. We used this fact ourselves to demonstrate that while commercial cerebral oximeters may agree well during normoxia, they may give very different values during hypoxia (Hessel et al. Acta Paediatr 2014;103:48893). I'll come back to that.

This increase in oxygen saturation more or less mirrors the change in upper-body systemic arterial saturation - from perhaps 70\% in the fetal state when 'breathing' through the placenta, to 95\% after birth when breathing by the lungs. So the data should be no surprise, and actually suggests that oxygen extraction is quite similar, so cerebral blood flow must be quite similar, so the 'hypoxia' - which is measured by NIRS as the hemoglobin-oxygen saturation in the vasculature and dominated by venous blood - is is not really reaching the cell populations that could crave for more supply.

But, the birth process is in between, and birth asphyxia is a constant threat and the point of departure for the present study. The authors focus on a bimodal distribution of the shape of the oxygenation, caracterized by slope of the rise and the time it took to reach the normal level: labelled the tortoise for the slow climb and the hare for the rapid. And this clearly was associated with blood gasses - the hares had lower $\mathrm{pH}$ and high lactate in the cord blood.

As all values of $\mathrm{pH}$ and Lactate were well within normal range, and Apgar scores were all 10, none of the infants would classify for a diagnosis of even mild birth asphyxia, so what we see must be classified as normal physiology. In this I may differ in my interpretation:

While the authors discuss 'strategies to protect the brain', I miss data on systemic oxygenation: ie. pulse oximetry and or heart rates. Since children who needed ventilation by mask was excluded, what we see is the result of the spontaneous initiation of breathing, aeration of the lungs, the opening of the pulmonary circulation and the reversal of fetal shunts. There is no data to describe these complexities, and an alternative explanation, more likely in my opinion, is that the 'physiologic acidosis' stimulated breathing after birth. In a similar study, using an instrument that integrates NIRS oximetry and flowmetry in a single 
probe, pulse oximetry was recorded in parallel (de Carli et al. Arch Dis Child. 2017;104:F648-53). This allowed calculation of the 'tissue oxygen extraction' which had much less variability, and - I assume - less bimodality.

The NIRS instrument used was the Hamamatzu NIRO200nx with $30 \mathrm{~mm}$ interoptode distance. This instrument performs quite well in the blood-lipid phantom, when compared to frequency domain oximetry, although underestimating the degree of hypoxia when hemoglobin concentration is low (Kleiser et al. Biomed Opt Exp 2017;9:86-101). Low hemoglobin is unlikely to be common in this group of healthy children and the authors should be complimented for contributing good data to the field. 